\title{
A rational belief: The method of discovery in the complex variable
}

\author{
Lorena Segura and Juan Matías Sepulcre
}

Department of Mathematical Analysis, University of Alicante, 03080-Alicante, Spain

\begin{abstract}
The importance of mathematics in the context of the scientific and technological development of humanity is determined by the possibility of creating mathematical models of the objects studied under the different branches of Science and Technology. The arithmetisation process that took place during the 19th Century consisted of the quest to discover a new mathematical reality in which the validity of logic would stand as something essential and central. Nevertheless, in contrast to this process, the development of mathematical analysis within a framework that largely involves intuition and geometry is a fact that cannot go unnoticed amongst the mathematics community, as we shall show in this paper through the research made by Bernhard Riemann on complex variables.
\end{abstract}

Keywords Mathematics - Complex variables - Arithmetisation - Method of discovery · Mathematical analysis

\section{Introduction}

The processes of the arithmetisation and rigorisation of mathematics during the 19th Century were largely intended to eliminate any reference to geometry and intuition that had predominated until then, and to emphasise the role of arithmetic and logic in the construction of new mathematical knowledge and its validation. As a result of those processes, more accurate fundamentals of logic and notion were obtained, erasing any trace of doubt on the consistency of the results obtained, and enhancing their rationale.

Even though the subject had been dealt with, the problems in the fundamentals of logic did not occupy a very prominent place amongst mathematicians until the beginning of the 19th Century, when evidence of elements of mathematics appeared to break the norm of coincidence in mathematics and nature. Also, from a theoretical point of view, the appearance of the quaternions, described by the Irish mathematician William Rowan Hamilton in 1843 (Hamilton 1844, 1853), and non-Euclidean geometry, on which several authors as János Bolyai and Nikolái Lobachevsky published treatises on hyperbolic geometry (Bonola 1954; Montesinos 1992), and who, in an attempt to prove Euclid's fifth postulate using a method of reduction to the absurd, demonstrated the existence of a new nature in mathematics as the established notions of intuition and construction could not explain those advances. Looking at the analysis, there was no rationale either in algebra or in the arithmetic used, and there were problems in geometry. Non-commutative quaternions and non-Euclidean geometry were what the historian Morris Kline (Kline 1980) described as an authentic disaster, considering them as the first disaster that would have extraordinary 
repercussions for the reflection on mathematics and for the evolution of the philosophy of mathematics.

Recent mathematical results showed the importance of structure and the validity of logic in respect of intuition understood as being related to sensitivity. For some, the fundamentals of mathematical construction are found precisely in the dimensions of logic and form, and in a drastic split from the concepts arising from intuition, visual geometry or the appeal to the empirical world, which some authors of the period considered to be contaminators of the origins of mathematics.

In this whole process there is an estrangement of these mechanisms of substantiation from the concepts and ideas that gave rise to calculus. However, it is not clear that mathematical construction can be limited to those dimensions of logic and that it can be unilaterally detached from intuition.

In this respect, throughout history, mathematics has been used in very diverse activities directly related with immediate space and physical reality. It has even been a device used by the priests of Mesopotamia for making predictions, or by the Pythagoreans expressing the harmony between divinity and human perfection, as a tool in the discipline of thought during the Middle Ages and also very useful in the search for answers from the universe from the Renaissance onwards. It has unquestionably been a key element in philosophical thought, amongst contemporary rationalists and philosophers. Nor can we fail to highlight its use as an instrument for creating works of art of great beauty, and as an aspect of entertainment (Boyer 1986).

In this paper we will defend that it has not been totally disassociated from intuition and geometrical perspective. In short, the development of analysis within a framework that is largely intuitive and geometrical is what is known as the discovery method, and through the work of Bernhard Riemann we will see how this method can be successful.

\section{Arithmetical perspective versus geometrical perspective}

The arithmetisation of the analysis and substantiation of calculus were immersed within a scenario that offered the specific evolution of new mathematics during the 19th Century. The emergence of non-Euclidean geometry or of abstract algebra (Keith 2012), and of a new nature to these disciplines, form part of this scenario in which a process of formalisation and axiomatisation of mathematics took place, with the participation of several important and distinguished mathematicians.

In spite of this, it cannot be said that a direct relationship existed between the creation of non-Euclidean geometry or the new algebras and the arithmetisation that took place during that century. In fact, some historians of mathematics analyse the role played by intuition in the development of non-Euclidean geometry, as it can be seen for example in (Gray 1979) and (Abardia, Reventós \& Rodríguez 2012). 
The new procedure based on an arithmetic perspective enjoyed great success which made any unhurried reflection on the soundness of its reasoning appear as secondary. It was a relevant point for the consolidation of logical deduction and rigour, as fundamental tools of mathematics or validation criteria perfectly assumed in those scientific communities.

The emphasis on algebraic and arithmetic demonstrations was a response to both the conceptual needs inherent to mathematics and to the needs of the mathematics community. Nevertheless, mathematics that were done during the 17th Century and earlier were based on intuition and the physical meaning, which is what, guided them. The reliability of its work did not lie in consistency or in formal rules, and therefore logic did not guide these discoveries. More so, the logic that was developed often left room for intuition and a sensible perspective of the outside world.

In the period prior to the arithmetisation process, the centre of mathematical analysis was calculus and, in spite of the enormous ignorance of logic and of the licentious use of numbers, this underwent a huge development. Irrational numbers were admitted at the beginning of the 19th Century, but not negative or complex numbers. This had great significance as was made evident in

Kline's quote (Kline 1980 p. 167): "A subtle change in the nature of mathematics had been unconsciously made by the masters. Up to about 1500 , the concepts of mathematics were immediate idealizations of or abstractions from experience (...) when irrational, negative, complex numbers, operations with letters, and the concepts of the derivate and integral entered mathematics, the subject became dominated by concepts derived from the recesses of human minds."

\section{An example of the method of discovery in the complex variable}

The modern theory of functions of a complex variable had several founders. Augustin-Louis Cauchy (1789-1857) and Carl Friedrich Gauss (1777-1855) were very important, but this section will specially focus on Karl Weierstrass (1815-1897) and Bernhard Riemann (1826-1866).

Weierstrass based his early work on the theory of series and the research done on elliptic and Abelian functions, established on solid foundations, in order to study the local representation of an analytic complex function as a series of powers on its convergence circle, as well as its corresponding analytic continuation. In fact, said local representation made him realise that an analytic function had many properties similar to those of a polynomial, which allowed him to consider the order of a zero and, through its development in series, prove the theorem on analytical solutions of a system of differential equations. $\mathrm{He}$ also introduced the notion of uniform convergence. In a nutshell, Weierstrass was characterised by the rigorous presentation of his theory and the careful development of his mathematical building, regardless of any reference to the geometric intuition. 
In contrast to Weierstrass' working method, we find Bernhard Riemann, a very versatile mathematician with a prolific mind, who took great interest in almost every aspect of mathematics. He made significant contributions to analysis and differential geometry, some of which paved the way for the later development of general relativity. His first publication was his doctoral dissertation "Grundlagen für eine allgemeine Theorie der Funktionen einer veränderlichen complexen Grösse" (Foundations for a general theory of functions of a complex variable) (Monastyrsky 2008, p.11). Particularly, he reached deep theorems that related the number theory to the classical analysis.

The distribution of prime numbers is, even today, a genuine unresolved mystery. Let $\pi(\mathrm{x})$ denote the amount of prime numbers less than or equal to $\mathrm{x}$. We know that the number of primes is infinite, hence $\pi(x) \rightarrow \infty$ when $x \rightarrow \infty$. $A$ more precise result was established by Leonhard Euler in 1737 (Ash \& Novinger 2007, p.149), when he proved that $\frac{1}{2}+\frac{1}{3}+\frac{1}{5}+\frac{1}{7}+\frac{1}{11}+\cdots=\infty$. Later, in 1749 , he found that

$\prod_{p}\left(1-p^{-x}\right)^{-1}=\sum_{n=1}^{\infty} n^{-x}, x>1$

where $p$ includes all prime numbers and $n$ all natural numbers. He even calculated the exact value for $x=2$, given by

$\sum_{n=1}^{\infty} \frac{1}{n^{2}}=\frac{\pi^{2}}{6}$.

This formula did not go unnoticed to Riemann, and it would mark the beginning of his research on the so-called Riemann zeta function, defined by

$\zeta(s):=\sum_{n=1}^{\infty} n^{-s}$,

where $s=\sigma+i t$ is a complex number. The series that defines $\zeta(s)$ converges absolutely on the half plane $\{s \in \mathrm{C}: \operatorname{Re}(s)>1\}$ and converges uniformly on compact subsets of $\{s \in \mathrm{C}: \operatorname{Re}(s) \geq 1+\delta\}$ for each $\delta>0$. Moreover, Euler's product formula is generalized as follows:

$\prod_{p}\left(1-p^{-s}\right)^{-1}=\sum_{n=1}^{\infty} n^{-s}$, Res $>1$.

On the other hand, $\zeta(s)$ has an analytic extension to $\{s \in \mathrm{C}: \operatorname{Re}(s)>0, s \neq 1\}$ and the above formula implies that $\zeta(s)$ has no zeros in the half plane $\{s \in \mathrm{C}: \operatorname{Re}(s)>1\}$, but Riemann was intrigued about the zeros of the extension of $\zeta(s)$ in $\{s \in \mathrm{C}: 0<\operatorname{Re}(s)<1\}$. Thus, after establishing the correspondence between the representation of the product and the function $\pi(\mathrm{x})$, and after studying some of the properties of $\zeta(s)$, Riemann stated in his seminal 1859 paper that he considered it "very likely" that all the zeros of $\zeta(s)$ in the above strip, $\{s \in \mathrm{C}: 0<\operatorname{Re}(s)<1\}$, called the critical strip, lie on the line $\operatorname{Re}(\mathrm{s})=\frac{1}{2}$. This assertion is now known as the Riemann hypothesis, and remains as yet unresolved (Ash \& Novinger 2007, p.149). 
The above is an example in which the geometric and intuitive reference was predominant in order to establish the connection of prime numbers to the known Riemann zeta function and to originate a subject that continues to capture the attention of eminent mathematicians. To Riemann, Euler's formula was really admirable, and it must be said that for the 100 years that passed between the discovery of the formula by Euler and Riemann's interest for it, the majority of mathematicians only considered it as a mere curiosity.

The name of Riemann is also linked to Riemann surfaces, which are generalizations of the complex plane to surfaces with several sheets in a way that a multi-valued function of a complex variable has only one value for each point on those surfaces. Riemann surfaces are one way of representing multiple-valued functions. Once the surface has been constructed for a given function, said function is single-valued on it and the single-valued functions theory can be applied. This way, the complications that arise due to the multi-valued nature of the function are avoided with a clever geometric resource. In this manner, the domain of analytic functions on the complex plane can be extended.

As per the above, the most striking aspect of Riemann's work can again be seen: the development of the analysis within a highly intuitive and geometric framework. In his report, and thanks to the role played by the geometric image in Bernhard Riemann's mathematical production, there were also several extension theorems for harmonic functions, the maximum modulus principle and the principle of analytic continuation. For Riemann, complex numbers were points of a plane and a complex function was a law by which surfaces can be transformed, so his aim was to represent and analyse these transformations.

In spite of this, Riemann himself admitted that his demonstrations were often incomplete, and that any potential gaps originated in their development could only be filled with the construction of new theories and mathematical entities.

\section{Conclusions}

The purpose to create mathematical models for the objects and the process studied in different branches of Technique and Science is very important in the value assigned to mathematics for the scientific and technological development of the humanity. In order to create a mathematical model, it is needed to have a deep and organized knowledge of real characteristics of the object to be studied. Therefore, improvements in a discipline are determined by the historical and cultural framework and this produces the boost of the progress of a branch in respect of the others. In this manner, for many years, the mathematical models were only used in Mechanics, Optical field, Astronomy and other branches of Physics, which were disciplines considered of special interest.

Most of current mathematical methods were appearing throughout history, motivated by requests received from other sciences in charge of investigating inorganic nature and, in particular, as was already mentioned on the previous paragraph, those dedicated to study the astronomic, mechanics and physicchemicals phenomenon, within the framework of the intuition. Later, it took 
place a generalization who has allowed the application of those mathematic methods to biological processes and, partially, to social ones, although application on social process has been more difficult. During the last decades of 20th century, investigations addressed to elaborate a mathematical method adequate for biological and social sciences have been increased.

One of the major problems that appears when we try to establish mathematical methods applicable to social sciences is the lack of exactness and precision of human thought and conducts. The blossom of a large variety of new branches of Mathematics, such as mathematical programming, mathematical systems theory, the mathematical theory of optimal control, stochastic processes theory, queuing theory, inventory theory, stochastic control theory, chaos or fractals theory, provides mathematical tools in order to define models for the problems, even more complex, emerged from the real need of its modelling and inspired in a strong intuitive framework. These branches reaffirm the need of the method of discovery as a key element in the mathematical production.

It follows from the above that mathematization of the science represents a double process of growth and development of concrete sciences and Mathematics itself. In other words, from necessity to ameliorate in other disciplines, Mathematics are forced to progress and, conversely, the progress of Mathematics with the introduction of new theories allows the possibility of applicability to other disciplines of the science. It can be observed that, sooner or later, all sciences start to use mathematical methods to a greater or lesser extent. The introduction of mathematics in all the fields of scientific knowledge is not a fortuitous episode in the development of Science and Technology and this process will be even wider and deeper in the future, with an eminently intuitive and geometrical framework.

In this regard we can find a quote from Morris Kline (Kline 1980): "The newly founded rigorous structure presumably guaranteed the soundness of mathematics but the guarantee was almost gratuitous. Not a theorem of arithmetic, algebra or Euclidean geometry was changed as a consequence, and the theorems of analysis had only to be more carefully formulated [...]. Indeed, the axioms had to yield the existing theorems rather than different ones because the theorems were on the whole correct. All of which means that mathematics rests not on logic but on sound intuition."

\section{Funding}

The authors was partially supported by Vicerrectorado de Investigación, Desarrollo e Innovación de la Universidad de Alicante under project GRE11-23.

\section{References}

- Abardia, J., Reventós, A. \& Rodríguez, C. J. (2012). What did Gauss read in the Appendix? Historia Mathematica 39, 292-323.

- Ash, R. B. \& Novinger, W. P. (2007). Complex Variables. New York: Dover Publications.

- Berkeley, G. (1734). The Analyst. London: J. Tonson. 
- Bonola, R. (1954). Non-Euclidean Geometry: A Critical and Historical Study of Its Development. New York: Dover Publications.

- Boyer, C. B. (1986). Historia de la matemática. Madrid: Alianza Editorial.

- Farkas, H. M. \& Kra, I. (1980). Riemann Surfaces. New York: SpringerVerlag.

- Gray, J., (1979). Non-Euclidean Geometry. A Re-interpretation. Historia Mathematica 6, 236-258.

- Hamilton, W. R. (1853). Lectures on Quaternions. Dublin: Hodges and Smith.

- Hamilton, W. R. (1844). On quaternions, or on a new system of imaginaries in algebra. Philosophical Magazine. 25 (3), 489-495.

- Keith, W. (2012). Introduction to Abstract Algebra. New Yersey: John Wiley \& Sons .

- Kline, M. (1980). Mathematics: The loss of certainty. Oxford: Oxford University Press.

- Monastyrsky, M. (2008). Riemann, Topology, and Physics. Boston: Birkhäuser.

- Montesinos, J. M. (1992); Las geometrías no euclídeas: Gauss, Lobachevski y Bolyai. Historia de la matemática en el siglo XIX. Madrid: Real Academía de Ciencias Exactas, Físicas y Naturales, 65-105.

\section{Biography}

L. Segura Abad graduated in 1994 in Mathematics from the Complutense University in Madrid. Since 1998, she has been working at the Department of Mathematical Analysis, University of Alicante. She was manager and speaker at courses and conferences of an educational nature. She is reviewer for some educational journals and author and coauthor of several teaching publications.

J.M. Sepulcre Martínez graduated in Mathematics and Statistical Sciences and Techniques. He received his Ph.D. in 2008 in Mathematics from the University of Alicante (Spain). Since 2007, he has been working at the Department of Mathematical Analysis, University of Alicante. He is author and coauthor of several research papers listed in MathSciNet, some of which have been published in top research journals, and several teaching publications. 